\title{
Impact of domain walls on the chiral magnetic effect in hot QCD matter
}

\author{
Kirill Tuchin \\ Department of Physics and Astronomy, Iowa State University, Ames, Iowa 50011, USA
}

(Received 26 March 2018; published 18 June 2018)

\begin{abstract}
The chiral magnetic effect (CME) - the separation of positive and negative electric charges along the direction of the external magnetic field in quark-gluon plasma and other topologically nontrivial media-is a consequence of the coupling of electrodynamics to the topological gluon field fluctuations that form metastable $C P$-odd domains. In phenomenological models it is usually assumed that the domains are uniform and the influence of the domain walls on the electric current flow is not essential. This article challenges the latter assumption. A simple model consisting of a uniform spherical domain in a uniform time-dependent magnetic field is introduced and analytically solved. It is shown that (i) no electric current flows into or out of the domain; (ii) the charge separation current, viz. the total electric current flowing inside the domain in the external field direction, is a dissipative Ohm current; (iii) the CME effect can be produced either by the anomalous current or by the boundary conditions on the domain wall; and (iv) the charge separation current oscillates in plasma long after the external field decays. These properties are qualitatively different from the CME in an infinite medium.
\end{abstract}

DOI: 10.1103/PhysRevC.97.064914

\section{INTRODUCTION}

The chiral magnetic effect (CME) is induction of electric current along the direction of the applied magnetic field [1-5]. It occurs in topologically nontrivial systems with chiral anomaly [6,7] and breaks local $P$ and $C P$ symmetries. A phenomenological manifestation of the CME is separation of positive and negative electric charges along the magnetic field direction [4]. In relativistic heavy-ion collisions, electric charges in quark-gluon plasma (QGP) separate along the direction of the external magnetic field created by the spectator valence quarks [4,8-17]. There are several phenomenological approaches that link this effect to the experimental data [18,19].

Quantitative analysis of the charge separation requires knowledge of the medium response to the external electromagnetic field. The simplest model is to add a new anomalous current $\boldsymbol{j}_{A}=\sigma_{\chi} \boldsymbol{B}$ to the Amper law, where the chiral conductivity $\sigma_{\chi}$ is assumed to be weakly dependent on position and time $[3,5,20]$. The time dependence of the chiral conductivity arises primarily due to the sphaleron transitions, the finite quark mass, and the helicity exchange between the magnetic field and QGP. All these effects have very long characteristic time scales compared to the QGP's lifetime [21-25], which justifies treating $\sigma_{\chi}$ as time independent. ${ }^{1}$ The assumption of the spatial uniformity is less sound however. The topological

Published by the American Physical Society under the terms of the Creative Commons Attribution 4.0 International license. Further distribution of this work must maintain attribution to the author(s) and the published article's title, journal citation, and DOI. Funded by $S C O A P^{3}$.

${ }^{1}$ Other, more exotic, effects that may induce time dependence are discussed in Refs. [26,27].
$C P$-odd fluctuations of the hot nuclear matter occupy a region of a typical size of $\sim 1 / g^{2} T$, which is of the order of a fm. This implies that a typical heavy-ion collision can produce a large number of topologically different metastable $C P$-odd domains. Electric current varies steeply between the domain interior and the surrounding plasma. Thus, the charge separation effect is expected to be strongly dependent on the domain size and topology. The main goal of this article is to compute the charge separation current taking into account these finite-size effects.

To study the charge separation effect in a finite-size domain, it is advantageous to consider an exactly solvable model. The model considered in this article consists of a spatially uniform spherical domain of radius $R$ immersed into a topologically trivial environment. The electrodynamics with the chiral anomaly is described by the Maxwell-Chern-Simons theory (MCS) in which the anomalous terms are associated with the background pseudoscalar field $\Theta$ whose dynamical extension is the axion [5,28-30]. The role of the chiral anomaly is twofold: it induces a new anomalous current into Ampere's law and causes a discontinuity of the normal electric and tangential magnetic field components at the domain wall even in the absence of the surface currents. Thus, the computation of the charge separation current entails solving the MCS equations inside the domain, in the presence of the anomalous current, and outside the domain and matching these solutions by means of the boundary conditions.

The article is structured as follows. The basic equations of the MCS theory and the corresponding boundary conditions are discussed in Sec. II. Considering a spatially uniform domain of an arbitrary shape, it is shown that the boundary conditions require vanishing of the normal component of the current on the domain wall. General solutions to the MCS equations inside and outside a domain are obtained in Sec. III A for a uniform monochromatic external field. Then in Sec. IIIB 
these solutions are matched using the boundary conditions that yield analytical expressions for the magnetic field spectrum in entire space. The result of Sec. IIIB allows one to compute the induced magnetic field for any time dependence of the external magnetic field. The analytical expressions for the total electric current flowing through any cross section of the domain perpendicular to the external field direction (31) and the magnetic moment of the domain are also derived. This is used in Sec. IV to numerically compute the magnetic field of the domain using the known time dependence of the external magnetic field produced in relativistic heavy-ion collisions. The results are summarized and discussed in Sec. V.

\section{FIELD EQUATIONS AND BOUNDARY CONDITIONS}

The field equations of electrodynamics coupled to the topological charge carried by the gluon field read [5,28-30]

$$
\begin{aligned}
\nabla \cdot \boldsymbol{B} & =0, \\
\nabla \cdot\left(\boldsymbol{E}+c_{A} \Theta \boldsymbol{B}\right) & =0, \\
\nabla \times \boldsymbol{E} & =-\partial_{t} \boldsymbol{B}, \\
\nabla \times\left(\boldsymbol{B}-c_{A} \Theta \boldsymbol{E}\right) & =\partial_{t}\left(\boldsymbol{E}+c_{A} \Theta \boldsymbol{B}\right)+\boldsymbol{j},
\end{aligned}
$$

where $c_{A}=N_{c} \sum_{f} q_{f}^{2} e^{2} / 2 \pi^{2}$ is the chiral anomaly coefficient. The plasma is assumed to be electrically neutral. The Ohm current is $\boldsymbol{j}=\sigma \boldsymbol{E}$, where $\sigma$ is the electrical conductivity. The background field $\Theta$ is regarded as spatially uniform everywhere except the domain wall where $\nabla \Theta$ is discontinuous.

As explained in the Introduction, the time variation of $\Theta$ is too slow to be important for the heavy-ion phenomenology. Nevertheless, because the chiral conductivity is proportional to the time derivative of $\Theta$ one needs to keep track of its small variations. Hence $\Theta$ is approximated by [25]

$$
\Theta \approx \Theta_{0}+\mu_{5} t
$$

where $\mu_{5}$ is the axial chemical potential related to the chiral conductivity $\sigma_{\chi}$ as $\mu_{5}=\sigma_{\chi} / c_{A}[3,5]$. Estimating the chiral conductivity optimistically as $\sigma_{\chi}=10^{-2} \mathrm{fm}^{-1}$ and using $c_{A}=$ $1 / 129$ one obtains $\mu_{5}=1.3 \mathrm{fm}^{-1}$. Thus, the time-dependent term in Eq. (2) is smaller than $2 \pi$ for $t<3 \mathrm{fm}$. From now on it is assumed that this condition is satisfied.

With the assumptions outlined in the preceding paragraphs one can simplify Eqs. (1a)-(1d), which read at any point in space except the domain wall, as follows:

$$
\begin{aligned}
\nabla \cdot \boldsymbol{B} & =0, \\
\nabla \cdot \boldsymbol{E} & =0, \\
\nabla \times \boldsymbol{E} & =-\partial_{t} \boldsymbol{B}, \\
\nabla \times \boldsymbol{B} & =\partial_{t} \boldsymbol{E}+\sigma_{\chi} \boldsymbol{B}+\boldsymbol{j} .
\end{aligned}
$$

The assumption of the uniformity of the domain interior means that its wall width is neglected. The boundary conditions on the domain wall can be obtained directly from Eqs. (1a)-(1d). Denoting by $\Delta$ the discontinuity of a field component across the domain wall and neglecting the time-dependent term in Eq. (2) one obtains [30]

$$
\begin{aligned}
\Delta B_{\perp} & =0, \\
\Delta\left(E_{\perp}+c_{A} \Theta_{0} B_{\perp}\right) & =0, \\
\Delta \boldsymbol{E}_{\|} & =0, \\
\Delta\left(\boldsymbol{B}_{\|}-c_{A} \Theta_{0} \boldsymbol{E}_{\|}\right) & =0,
\end{aligned}
$$

where $E_{\perp}, B_{\perp}$ and $\boldsymbol{E}_{\|}, \boldsymbol{B}_{\|}$are components of the electromagnetic field normal and tangential to the domain wall, respectively.

A more stringent boundary condition can be derived using the continuity equation $\nabla \cdot \boldsymbol{j}=0$, which implies that $\Delta j_{\perp}=0$ [31]. Projecting Eq. (1d) onto the normal direction and using Eq. (1c) one obtains

$$
\begin{aligned}
& (\nabla \times \boldsymbol{B})_{\perp}+c_{A} \Theta \partial_{t} B_{\perp}-c_{A}(\nabla \Theta \times \boldsymbol{E})_{\perp} \\
& \quad=\partial_{t}\left(E+c_{A} \Theta B\right)_{\perp}+j_{\perp} .
\end{aligned}
$$

The third term on the left-hand side vanishes because $\nabla \Theta$ points in the normal direction. The terms on the right-hand side are continuous in view of Eq. (4b). Now, the solution of Eq. (3d) is a complete set of eigenstates of the curl operator satisfying the equation $\nabla \times \boldsymbol{B}=\alpha \boldsymbol{B}$, where $\alpha$ depends on medium properties. Consider such an eigenstate of frequency $\omega$. Then Eq. (5) implies that $B_{\perp}\left(\alpha+i \omega c_{A} \Theta\right)$ is continuous across the wall. However, $B_{\perp}$ is also continuous, whereas $\alpha$ and $\Theta$ are discontinuous. These conditions can only be satisfied if $B_{\perp}$ vanishes on the wall:

$$
\left.B_{\perp}\right|_{\text {wall }}=0 .
$$

\section{ELECTROMAGNETIC FIELD OF A SPHERICAL DOMAIN IN A UNIFORM MONOCHROMATIC MAGNETIC FIELD}

\section{A. General solution inside and outside the domain}

The external homogeneous magnetic field of frequency $\omega$ induces the electromagnetic field in the domain that is governed by Eqs. (3) and boundary conditions (4) and (6). Because electric and magnetic fields are divergentless, it is convenient to use the radiation gauge $\nabla \cdot \boldsymbol{A}=0, A^{0}=0$, which allows one to write Eq. (3d) as an equation for the vector potential:

$$
\nabla^{2} \boldsymbol{A}=\partial_{t}^{2} \boldsymbol{A}+\sigma \partial_{t} \boldsymbol{A}-\sigma_{\chi} \boldsymbol{\nabla} \times \boldsymbol{A} .
$$

Separation of the temporal dependence of the vector-potential $\boldsymbol{A}(\boldsymbol{x}, t)=\boldsymbol{A}_{\omega}(\boldsymbol{x}) e^{-i \omega t}$ yields the following for its monochromatic component:

$$
\nabla^{2} \boldsymbol{A}_{\omega}=-\omega(\omega+i \sigma) \boldsymbol{A}_{\omega}-\sigma_{\chi} \nabla \times \boldsymbol{A}_{\omega} .
$$

The general solution of Eq. (8) can be written as a superposition of the eigenfunctions of the curl operator. These functions are denoted by $\boldsymbol{W}_{l m}^{ \pm}(\boldsymbol{x}, \alpha)$ and satisfy the equation

$$
\nabla \times \boldsymbol{W}_{l m}^{ \pm}(\boldsymbol{x}, \alpha)= \pm \alpha \boldsymbol{W}_{l m}^{ \pm}(\boldsymbol{x}, \alpha)
$$

Their explicit form in the spherical coordinates reads [31]

$$
\boldsymbol{W}_{l m}^{ \pm}(\boldsymbol{x}, \alpha)=\boldsymbol{T}_{l m}(\boldsymbol{x}, \alpha) \mp i \boldsymbol{P}_{l m}(\boldsymbol{x}, \alpha),
$$

where

$$
\boldsymbol{T}_{l m}(\boldsymbol{x}, \alpha)=\frac{f_{l}(\alpha r)}{\sqrt{l(l+1)}}\left\{-\frac{m}{\sin \theta} Y_{l}^{m}(\theta, \phi) \hat{\boldsymbol{\theta}}-i \partial_{\theta} Y_{l}^{m}(\theta, \phi) \hat{\boldsymbol{\phi}}\right\},
$$




$$
\begin{aligned}
\boldsymbol{P}_{l m}(\boldsymbol{x}, \alpha)= & \frac{1}{\sqrt{l(l+1)}}\left\{-\frac{l(l+1)}{\alpha r} f_{l}(\alpha r) Y_{l}^{m}(\theta, \phi) \hat{\boldsymbol{r}}\right. \\
& -\frac{1}{\alpha r} \partial_{r}\left[f_{l}(\alpha r) r\right] \partial_{\theta} Y_{l}^{m}(\theta, \phi) \hat{\boldsymbol{\theta}} \\
& \left.-\frac{i m}{\alpha \sin \theta} f_{l}(\alpha r) Y_{l}^{m}(\theta, \phi) \hat{\boldsymbol{\phi}}\right\} .
\end{aligned}
$$

$f_{l}$ is a linear combination of the spherical Bessel functions $j_{l}$ and $n_{l}$. The $z$ axis is chosen in the direction of the external magnetic field, which is given by

$$
\boldsymbol{B}^{\mathrm{ext}}=B_{0} \hat{z} e^{-i \omega t}=B_{0}(\cos \theta \hat{\boldsymbol{r}}-\sin \theta \hat{\boldsymbol{\theta}}) e^{-i \omega t} .
$$

The corresponding vector potential is

$$
\boldsymbol{A}^{\mathrm{ext}}=\frac{1}{2} B_{0} r \sin \theta \hat{\boldsymbol{\phi}} e^{-i \omega t} .
$$

The symmetry considerations imply that in a spherical domain the only nontrivial component of the induced field is proportional to the linear combination of the functions:

$$
\begin{aligned}
\boldsymbol{W}_{10}^{ \pm}(\boldsymbol{x}, \alpha)= & -\frac{i}{\sqrt{2}} f_{1}(\alpha r) \partial_{\theta} Y_{1}^{0}(\theta, \phi) \hat{\boldsymbol{\phi}} \\
& \pm \frac{i \sqrt{2}}{\alpha r} f_{1}(\alpha r) Y_{1}^{0}(\theta, \phi) \hat{\boldsymbol{r}} \\
& \pm \frac{i}{\sqrt{2} \alpha r} \partial_{r}\left[f_{1}(\alpha r) r\right] \partial_{\theta} Y_{1}^{0}(\theta, \phi) \hat{\boldsymbol{\theta}} .
\end{aligned}
$$

The general solution to Eq. (8) inside the domain reads

$$
\boldsymbol{A}_{\omega}^{\mathrm{in}}(\boldsymbol{x})=\sum_{l m}\left[g_{l m} \boldsymbol{W}_{l m}^{+}\left(\boldsymbol{x}, q_{+}\right)+h_{l m} \boldsymbol{W}_{l m}^{-}\left(\boldsymbol{x}, q_{-}\right)\right],
$$

where $q_{ \pm}$are the roots of the equations $-q_{ \pm}^{2}=-\omega(\omega+i \sigma) \mp$ $\sigma_{\chi} q_{ \pm}$. Namely, ${ }^{2}$

$$
q_{ \pm}= \pm \frac{\sigma_{\chi}}{2}+\sqrt{\left(\sigma_{\chi} / 2\right)^{2}+\omega(\omega+i \sigma)} .
$$

The boundary conditions at the origin require that $f_{l}\left(q_{ \pm} r\right)=$ $j_{l}\left(q_{ \pm} r\right)$. In view of Eq. (9), the magnetic field inside the domain is

$$
\boldsymbol{B}_{\omega}^{\mathrm{in}}(\boldsymbol{x})=\sum_{l m}\left[g_{l m} q_{+} \boldsymbol{W}_{l m}^{+}\left(\boldsymbol{x}, q_{+}\right)-h_{l m} q_{-} \boldsymbol{W}_{l m}^{-}\left(\boldsymbol{x}, q_{-}\right)\right]
$$

The general solution to Eq. (7) outside the domain, where $\Theta=0$, reads

$$
\boldsymbol{A}_{\omega}^{\text {out }}(\boldsymbol{x})=\sum_{l m}\left[c_{l m} \boldsymbol{W}_{l m}^{+}(\boldsymbol{x}, k)+d_{l m} \boldsymbol{W}_{l m}^{-}(\boldsymbol{x}, k)\right],
$$

where $k=\sqrt{\omega(\omega+i \sigma)}$ and $f_{l}(k r)=\cos \delta_{l} j_{1}(k r)-\sin \delta_{l} n_{l}(k r)$. The magnetic field outside the domain is

$$
\boldsymbol{B}_{\omega}^{\text {out }}(\boldsymbol{x})=\sum_{l m} k\left[c_{l m} \boldsymbol{W}_{l m}^{+}(\boldsymbol{x}, k)-d_{l m} \boldsymbol{W}_{l m}^{-}(\boldsymbol{x}, k)\right] .
$$

Note that Eqs. (19) and (20) do not include the external field.

\footnotetext{
${ }^{2}$ The other two roots give linearly dependent solutions. They can be obtained by replacing $q_{ \pm} \rightarrow-q_{\mp}$ which corresponds to $T_{l m} \rightarrow(-1)^{l} T_{l m}, P_{l m} \rightarrow(-1)^{l+1} P_{l m}$.
}

\section{B. Matching the solutions on the domain wall}

The boundary conditions (4) and (6) on the spherical domain wall of radius $R$ read, after replacing $\boldsymbol{E}_{\omega}=i \omega \boldsymbol{A}_{\omega}$, as follows:

$$
\begin{aligned}
\left.B_{\omega r}^{\text {in }}\right|_{r=R} & =\left.B_{\omega r}^{\text {out }}\right|_{r=R}+B_{0} \cos \theta=0, \\
\left.A_{\omega r}^{\text {in }}\right|_{r=R} & =\left.A_{\omega r}^{\text {out }}\right|_{r=R}, \\
\left.A_{\omega \theta}^{\text {in }}\right|_{r=R} & =\left.A_{\omega \theta}^{\text {out }}\right|_{r=R}, \\
\left.A_{\omega \phi}^{\text {in }}\right|_{r=R} & =\left.A_{\omega \phi}^{\text {out }}\right|_{r=R}+\frac{1}{2} B_{0} R \sin \theta \\
\left.\left(B_{\omega \theta}^{\text {in }}+i \omega c_{A} \Theta_{0} A_{\omega \theta}^{\text {in }}\right)\right|_{r=R} & =\left.B_{\omega \theta}^{\text {out }}\right|_{r=R}-B_{0} \sin \theta \\
\left.\left(B_{\omega \phi}^{\text {in }}+i \omega c_{A} \Theta_{0} A_{\omega \phi}^{\text {in }}\right)\right|_{r=R} & =\left.B_{\omega \phi}^{\text {out }}\right|_{r=R} .
\end{aligned}
$$

Because the external magnetic field can be written as $\boldsymbol{B}_{\omega}^{\text {ext }}=$ $-\sqrt{6 \pi} B_{0} \boldsymbol{P}_{10}(\boldsymbol{x}, 0)$, the only nontrivial solution to Eq. (21) is for the partial amplitudes with $l=1$ and $m=0$. It is easy to verify, using Eqs. (16), (18), (19), and (20), that the boundary conditions (21a) and (21d) are identical. Also, vanishing of $B_{\omega r}^{\text {in }}$ on the wall, i.e., Eq. (21a), implies vanishing of $A_{\omega \phi}$ on the wall, which in turn indicates that Eqs. (21b) and (21f) are identical. Thus, there are five equations to determine five unknown amplitudes: $g_{10}, h_{10}, c_{10}, d_{10}$, and $\delta_{1}$. It is understood that $\Theta \neq 0$ inside the domain because otherwise some of Eqs. (21a)-(21f) become redundant.

To write the solution of the boundary conditions (21) in a compact form denote $\left.\partial_{r}\left[j_{1}(\alpha r) r\right]\right|_{r=R} \equiv\left[j_{1}(\alpha R) R\right]^{\prime}$ and define the following three auxiliary functions:

$$
\begin{aligned}
& W_{1}=j_{1}\left(R q_{+}\right)\left[j_{1}\left(R q_{-}\right) R\right]^{\prime}-j_{1}\left(R q_{-}\right)\left[j_{1}\left(R q_{+}\right) R\right]^{\prime}, \quad(22 \mathrm{a}) \\
& W_{2}=j_{1}\left(R q_{+}\right)\left[j_{1}\left(R q_{-}\right) R\right]^{\prime} q_{+}+j_{1}\left(R q_{-}\right)\left[j_{1}\left(R q_{+}\right) R\right]^{\prime} q_{-},
\end{aligned}
$$

$$
W_{3}=j_{1}(R k)\left[n_{1}(R k) R\right]^{\prime}-n_{1}(R k)\left[j_{1}(R k) R\right]^{\prime} .
$$

After tedious but straightforward algebraic manipulations one obtains

$$
\begin{aligned}
g_{10} & =\sqrt{\frac{2 \pi}{3}} B_{0} R \frac{k^{2}}{\omega} \frac{W_{2}-2\left(q_{+}+q_{-}\right) j_{1}\left(R q_{+}\right) j_{1}\left(R q_{-}\right)}{j_{1}\left(R q_{+}\right)\left(q_{+}+q_{-}\right)\left[\frac{i k^{2}}{\omega} W_{1}+c_{A} \Theta W_{2}\right]} \\
h_{10} & =-\frac{j_{1}\left(R q_{+}\right)}{j_{1}\left(R q_{-}\right)} g_{10} .
\end{aligned}
$$

Equation (24) follows directly from the boundary condition (6), or equivalently, Eq. (21a). Other amplitudes can be expressed in terms of $g_{10}$. Define two more auxiliary functions:

$$
\begin{aligned}
& a=g_{10} \frac{q_{+}+q_{-}}{k} j_{1}\left(R q_{+}\right), \\
& b=-\frac{g_{10}}{j_{1}\left(R q_{-}\right)}\left[W_{1}+W_{2}\left(\frac{1}{k}+\frac{i c_{A} \Theta \omega}{k^{2}}\right)\right] .
\end{aligned}
$$


The amplitudes of the positive helicity component of the magnetic field outside the domain, see Eq. (20), are

$$
\begin{aligned}
c_{10} \cos \delta_{1}= & -\frac{1}{2 W_{3}}\left\{\left(b+i R B_{0} 2 \sqrt{2 \pi / 3}\right) n_{1}(R k)\right. \\
& \left.-\left(a+i R B_{0} \sqrt{2 \pi / 3}\right)\left[n_{1}(R k) R\right]^{\prime}\right\}, \\
-c_{10} \sin \delta_{1}= & \frac{1}{2 W_{3}}\left\{\left(b+i R B_{0} 2 \sqrt{2 \pi / 3}\right) j_{1}(R k)\right. \\
& \left.-\left(a+i R B_{0} \sqrt{2 \pi / 3}\right)\left[j_{1}(R k) R\right]^{\prime}\right\},
\end{aligned}
$$

The ratio of these equations immediately yields $\tan \delta_{1}$. The remaining amplitudes, corresponding to the negative helicity component of the magnetic field outside the domain, read

$$
\begin{aligned}
d_{10} \cos \delta_{1} & =\frac{i R B_{0} \sqrt{2 \pi / 3}-a}{2\left[j_{1}(R k)-\tan \delta_{1} n_{1}(R k)\right]}, \\
-d_{10} \sin \delta_{1} & =\frac{i R B_{0} \sqrt{2 \pi / 3}-a}{2\left[-\cot \delta_{1} j_{1}(R k)+n_{1}(R k)\right]} .
\end{aligned}
$$

Substitution of Eqs. (22a)-(26d) into Eqs. (18) and (20) furnishes the analytic expressions for the electromagnetic field of the spherical domain in the monochromatic uniform magnetic field.

\section{Electric current and magnetic moment}

Using the results of the previous section one can compute the total current flowing in the direction of the external magnetic field through any cross sectional area of the domain:

$$
I_{\omega}=\sigma_{\chi} \int B_{\omega z} d S_{z}+\sigma \int E_{\omega z} d S_{z}=\sigma_{\chi} \Phi_{B}+\sigma \Phi_{E} .
$$

The magnetic field flux can be written as

$$
\begin{aligned}
\Phi_{B} & =2 \pi \sigma_{\chi} \int_{0}^{\sqrt{R^{2}-z^{2}}} B_{\omega z} \rho d \rho \\
& =2 \pi \sigma_{\chi} \int_{0}^{\sqrt{R^{2}-z^{2}}}\left(\cos \theta B_{\omega r}-\sin \theta B_{\omega \theta}\right) \rho d \rho \\
& =2 \pi \sigma_{\chi} \int_{z}^{R}\left(\frac{z}{r} B_{\omega r}-\frac{\rho}{r} B_{\omega \theta}\right) r d r,
\end{aligned}
$$

where $\rho$ is the radial coordinate in the cross-sectional plane and in the second line the integration variable has been changed to $r=\sqrt{\rho^{2}+z^{2}}$. Using Eqs. (18) and (15) one derives

$$
\begin{aligned}
\Phi_{B}= & 2 \pi\left\{z^{2} i \sqrt{\frac{3}{2 \pi}} \int_{z}^{R} \frac{d r}{r^{2}}\left[g_{10} j_{1}\left(q_{+} r\right)+h_{10} j_{1}\left(q_{-} r\right)\right]\right. \\
& -\frac{i}{2} \sqrt{\frac{3}{2 \pi}} \int_{z}^{R} \frac{d r}{r^{2}}\left(r^{2}-z^{2}\right) \\
& \left.\times\left\{g_{10}\left[j_{1}\left(q_{+} r\right) r\right]^{\prime}+h_{10}\left[j_{1}\left(q_{-} r\right) r\right]^{\prime}\right\}\right\} .
\end{aligned}
$$

Integrating the second integral by parts and using the boundary condition (24) yields

$$
\Phi_{B}=0
$$

Thus, the anomalous component of the current does not contribute to the charge separation current.

The computation of the electric flux can be done along the same lines by noting that $E_{\omega z}=i \omega A_{\omega z}$ and using Eq. (16) in place of Eq. (18). The result is

$$
I_{\omega}=\sigma \Phi_{E}=-\sigma \omega \sqrt{\frac{3 \pi}{2}} \frac{R^{2}-z^{2}}{R} j_{1}\left(R q_{+}\right)\left(q_{+}+q_{-}\right) g_{10} .
$$

This constitutes the charge separation effect. The current $I_{\omega}$ does not identically vanish as long as $\Theta \neq 0$; i.e., either $\Theta_{0}$ or $\sigma_{\chi}$ is finite.

The magnetic moment of the domain is given by ${ }^{3}$

$$
\boldsymbol{\mu}=\frac{1}{2} \sigma_{\chi} \int \boldsymbol{x} \times \boldsymbol{B} d^{3} x+\frac{1}{2} \sigma \int \boldsymbol{x} \times \boldsymbol{E} d^{3} x
$$

and can be computed using the same steps as were employed in the calculation of the current. The result is

$$
\begin{aligned}
\boldsymbol{\mu}_{\omega}= & i \hat{z} \sqrt{\frac{2 \pi}{3}}\left\{\frac{g_{10}}{q_{+}^{3}}\left[\left(3-R^{2} q_{+}^{2}\right) \sin \left(R q_{+}\right)-3 R q_{+} \cos \left(R q_{+}\right)\right]\right. \\
& \times\left(\sigma_{\chi}+\frac{i \omega \sigma}{q_{+}}\right) \\
& -\frac{h_{10}}{q_{-}^{3}}\left[\left(3-R^{2} q_{-}^{2}\right) \sin \left(R q_{-}\right)-3 R q_{-} \cos \left(R q_{-}\right)\right] \\
& \left.\times\left(\sigma_{\chi}-\frac{i \omega \sigma}{q_{-}}\right)\right\} .
\end{aligned}
$$

It vanishes if $\sigma_{\chi} \rightarrow 0$; i.e., the existence of the domain magnetic moment requires the anomalous current.

\section{APPLICATION TO HEAVY-ION COLLISIONS}

In this section we specialize the results of the previous section to the heavy-ion collisions phenomenology. The quarkgluon plasma produced in heavy-ion collisions is subject to the external magnetic field induced by the spectator valence charges [4,8-17]. The time dependence of this field is quite complicated. It is convenient to adopt a simple parametrization introduced in Refs. [19,33]:

$$
\boldsymbol{B}^{\operatorname{ext}}(t)=\frac{B_{0} \hat{z}}{1+\left(t / t_{0}\right)^{2}}=\frac{1}{2} B_{0} t_{0} \hat{z} \int_{-\infty}^{\infty} d \omega e^{-t_{0}|\omega|-i \omega t},
$$

where $t_{0}=0.6 \mathrm{fm}$. It accounts for the fact that an electrically conducting medium slows down the decay of the electromagnetic field [16,17,34-36]. The magnetic field inside the domain follows from Eq. (18):

$$
\begin{aligned}
\boldsymbol{B}^{\text {in }}(\boldsymbol{x}, t)= & \frac{1}{2} t_{0} \hat{z} \int_{-\infty}^{+\infty} d \omega e^{-t_{0}|\omega|-i \omega t} \\
& \times\left[g_{10} q_{+} \boldsymbol{W}_{10}^{+}\left(\boldsymbol{x}, q_{+}\right)-h_{10} q_{-} \boldsymbol{W}_{10}^{-}\left(\boldsymbol{x}, q_{-}\right)\right] .
\end{aligned}
$$

General properties of the magnetic-field time dependence can be inferred from the analytical structure of its Fourier component. The amplitudes $g_{10}$ and $h_{10}$ have poles at $R q_{+}=x_{n}$ and

\footnotetext{
${ }^{3}$ The domain magnetization in the presence of anomalous currents has been previously discussed in Ref. [32].
} 

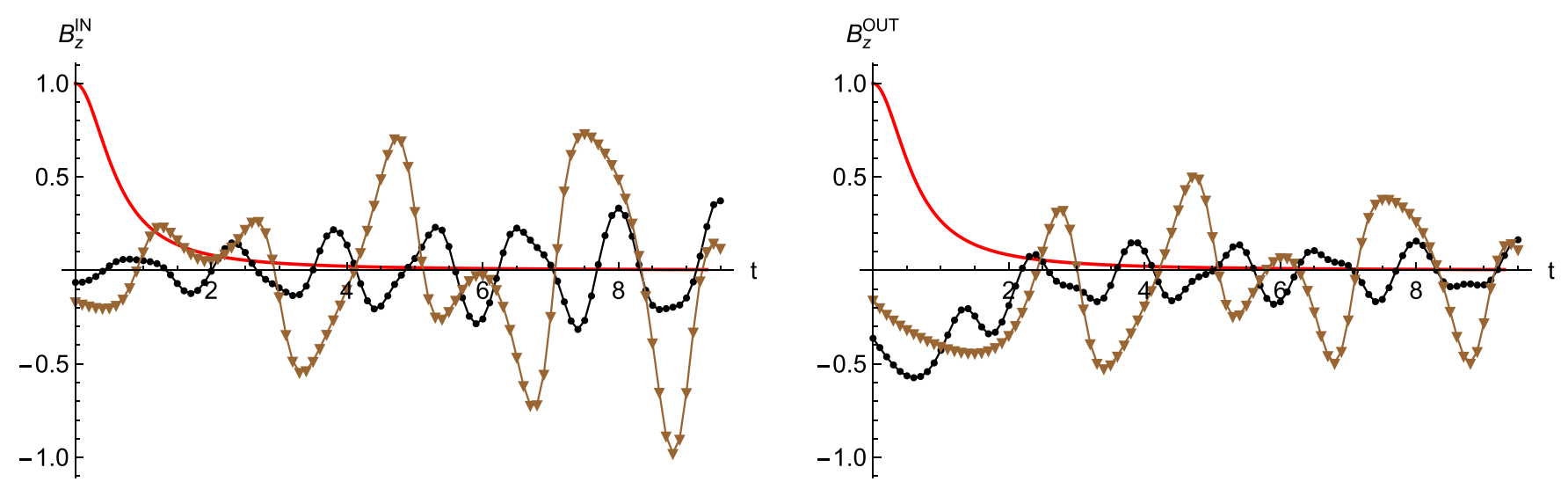

FIG. 1. Left panel: $B_{z}$ inside a spherical domain at a representative point $r=R / 2, \theta=\pi / 3$. Right panel: $B_{z}$ outside the domain at a representative point $r=3 R / 2, \theta=\pi / 3$. The domain radius is $R=1 \mathrm{fm}$ (black dots) or $R=2 \mathrm{fm}$ (brown triangles). Other parameters: $B_{0}=1 \mathrm{fm}^{-2}, \sigma=1 /(36 \mathrm{fm})[37-41], \sigma_{\chi}=1 /(100 \mathrm{fm})$, and $\Theta_{0}=2 \pi$. Solid red line represents the external field $B^{\text {ext }}$ of Eq. (34).

$R q_{-}=x_{n}$ correspondingly, where $x_{n}, n=0,1,2 \ldots$, are zeros of the spherical Bessel function $j_{1}(x)$. The first three zeros are $x_{1}=4.49, x_{2}=7.73$, and $x_{3}=10.90$. The characteristic external field frequency $\omega_{0} \sim 1 / t_{0}=1.7 \mathrm{fm}^{-1}$ is much larger than $\sigma$ and $\sigma_{\chi}$, which implies that the poles of $B_{\omega}^{\text {in }}$ are situated at $\omega \approx x_{n} / R$. Depending on the domain radius $R$ the integral over $\omega$ may pick up contributions from one or more poles. If $R<x_{1} / \omega_{0}=2.6 \mathrm{fm}$, which is the phenomenologically most relevant case, the magnetic field inside the domain is suppressed by the factor $e^{-t_{0} x_{1} / R}$. The magnetic fields of domains with sizes $2.6<R<4.6 \mathrm{fm}$ have the nonsuppressed contributions of the first zero, while contributions of other zeros are still exponentially suppressed.

This analysis is corroborated by the numerical calculation shown in Fig. 1. It is seen that the induced field strength increases with the domain radius. It is worth noticing that even though the initial field decays at about $2 \mathrm{fm}$, the induced field oscillates long after that time due to the low electrical conductivity of the QGP. Actually, the oscillation amplitude of the magnetic field inside the domain increases, indicating instability. This instability is caused by the brunch cut singularity along the imaginary axis in the expression for $B_{\omega}^{\text {in }}$ :

$$
\frac{i}{2}\left(-\sigma-\sqrt{\sigma^{2}+\sigma_{\chi}^{2}}\right) \leqslant \omega \leqslant \frac{i}{2}\left(-\sigma+\sqrt{\sigma^{2}+\sigma_{\chi}^{2}}\right) .
$$

This instability has been a subject of intensive study in recent years [25,42-58]. It is established that the growth of this instability is governed by the chiral anomaly equation. The unstable modes transfer helicity from the medium to the field in a process known as the inverse cascade [43,59]. Eventually, however, the helicity conservation puts a cap on the inverse cascade [60,61]. As explained in Sec. II, this interesting effect is not really phenomenologically relevant for heavy-ion collisions. In fact, Eq. (2) explicitly neglects any significant long-time evolution effects.

One can get a general idea about the magnetic field structure inside a spherical domain by looking at the snapshot shown in Fig. 2. As can be expected, the field lines are mostly twisted around the direction of the external field due to the smallness of the anomalous current. To better see the $z$ component of
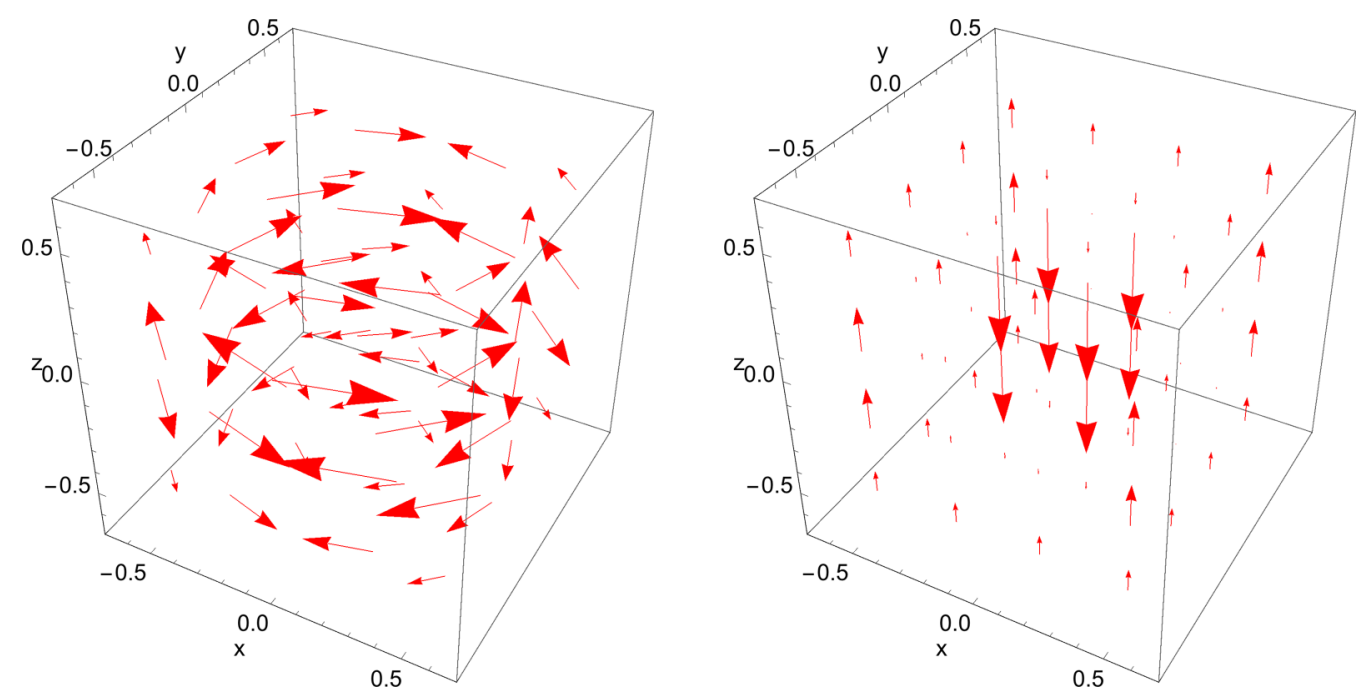

FIG. 2. Snapshot of the magnetic field inside a spherical domain of radius $R=1 \mathrm{fm}$ at $t=2 \mathrm{fm}$. Left panel: $\boldsymbol{B}$, right panel: $B_{z}$ (zoomed in). Other parameters: $B_{0}=1 \mathrm{fm}^{-2}, \sigma=1 /(36 \mathrm{fm}), \sigma_{\chi}=1 /(100 \mathrm{fm}), \Theta_{0}=2 \pi$. 

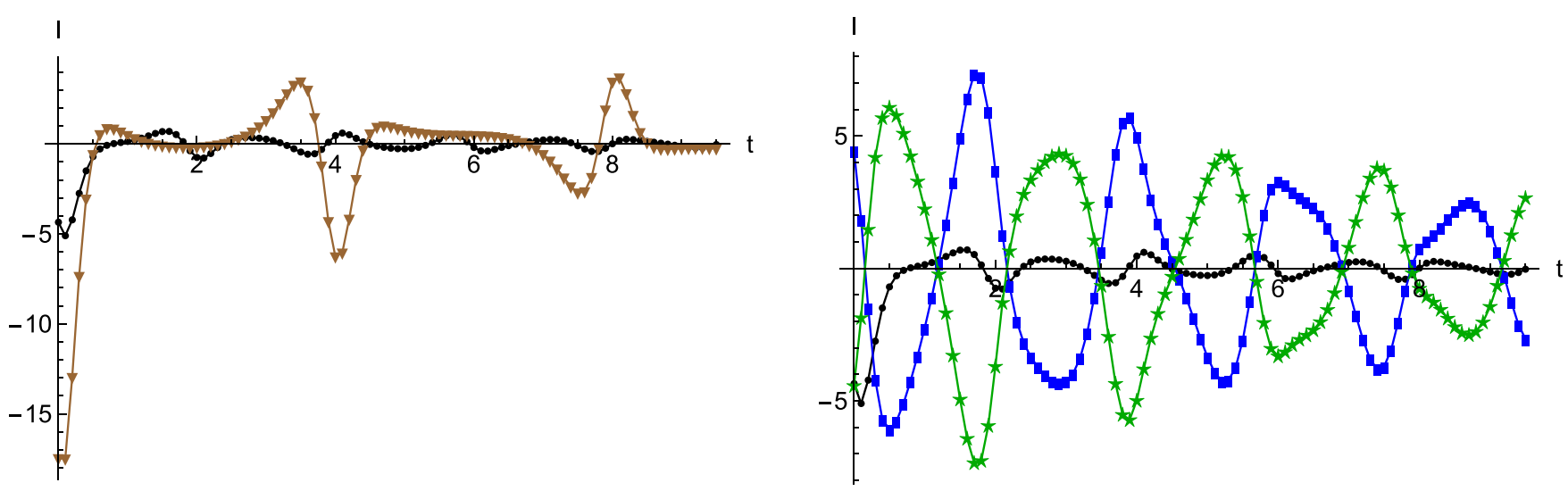

FIG. 3. Electric current flowing inside a domain in the $z$ direction through a cross section at $z=R / 2 . B_{0}=1 \mathrm{fm}^{-2}$, and $\sigma=1 /(36 \mathrm{fm})$. Black circles: $R=1 \mathrm{fm}, \sigma_{\chi}=0.01 / \mathrm{fm}$, and $\Theta_{0}=2 \pi$. Brown triangles: $R=2 \mathrm{fm}, \sigma_{\chi}=0.01 / \mathrm{fm}$, and $\Theta_{0}=2 \pi$. Blue squares: $R=1 \mathrm{fm}$, $\sigma_{\chi}=0.01 / \mathrm{fm}$, and $\Theta_{0}=-2 \pi$. Green stars: $R=1 \mathrm{fm}, \sigma_{\chi}=-0.01 / \mathrm{fm}$, and $\Theta_{0}=2 \pi$.

the magnetic field, the right panel of Fig. 2 magnifies it while discarding the transverse components. As has been shown in Sec. III B, the magnetic field flux through the cross-sectional area of the domain parallel to the $x y$ plane vanishes. As the result, the number of magnetic field lines crossing in and out of any $x y$ plane is equal. This can be seen in the right panel of Fig. 2 as well.

Even though the magnetic field does not produce net electric current in the $z$ direction, the electric current does. The induced electric current inside the domain is displayed in Fig. 3 for a representative set of phenomenologically relevant parameters. One observes rapid oscillations of the current that may average to zero in a long run. Also, at any given time, an average value of the total current of a large enough ensemble of domains seems to average to zero.

It would be interesting to investigate the behavior of the magnetic field and the induced current in the limit of large domain size $R \gg 1 \mathrm{fm}$. It was argued in Refs. [62,63] that the hot nuclear matter exhibits certain long-range correlations that ought be clearly seen in this limit. Unfortunately, the poor convergence of my numerical procedure at large $R$, due to the proliferation of the Bessel function zeros, did not allow me to verify the predictions of Refs. [62,63].

\section{SUMMARY AND DISCUSSION}

Metastable $C P$-odd topological domains emerge in the hot QCD matter. The external magnetic field applied to these domains generates an anomalous current and charge densities. This article focused on one such domain. To simplify the calculations, the domain was assumed to be a uniform sphere, while the surrounding medium was assumed to be spatially uniform and topologically trivial. The electromagnetic field in the entire space was analytically calculated by employing a standard technique. The electric and magnetic components of the field induce Ohm and anomalous currents, respectively. Their main properties are as follows.

(i) The normal component of the electric current vanishes on the domain wall regardless of the domain geometry and uniformity. Thus no electric current flows into or out of the domain. (ii) The charge separation current, i.e., the total electric current flowing in the direction of the external magnetic field through any cross sectional area of the domain is the Ohm current, as shown in Sec. IIIC. The contribution of the total anomalous current is zero. In particular, the total current vanishes in an electric insulator $\sigma \rightarrow 0$. This may appear counterintuitive because a $C P$-odd effect cannot be generated by the $C P$-even current. There is no contradiction though, because the the total current vanishes when $\Theta \rightarrow 0$. Even so, it is interesting to note that the current is finite if either $\Theta_{0}$ or $\sigma_{\chi}$ is finite. This is especially important if $\sigma_{\chi}$ turns out to be much smaller than a few $\mathrm{MeV}$ as assumed in most applications; in that case the CME is generated by the domain walls.

(iii) The total current is finite long after the external field has decayed, due to the low electrical conductivity of QGP, which implies small dissipation. The current oscillates with roughly the characteristic time $t_{0}$ of the external field. However, because no charge leaves the domain, the final charge separation within the domain depends on the current magnitude and direction at the time of the freeze-out.

(iv) The resonance frequencies of a spherical domain are $\omega_{n}=x_{n} / R$, where $x_{n}$ are zeros of the spherical Bessel function $j_{1}(x)$. The current frequency modes with $\omega \ll$ $\omega_{1}$ do not contribute to the total current because the corresponding wavelength does not fit in the domain. In the static limit $I_{\omega} \rightarrow 0$ as $\omega \rightarrow 0 .^{4}$

Finally, I believe that the present model, despite its simplicity, gives a reasonably accurate idea about a possible effect of the domain size on the charge separation effect. It has been seen throughout the article that the properties enumerated above are

\footnotetext{
${ }^{4}$ Actually, the MCS equations do have nontrivial solutions even in the absence of the external field. These are given by the Chandrasekhar-Kendall states, Eqs. (10)-(12), with $\alpha=\sigma_{\chi}$. However, their radii $R_{n}=x_{n} / \sigma_{\chi}$ are way too big to fit into the QGP, as was first pointed out in Ref. [64].
} 
fairly geometry independent. The gradients $\nabla \Theta$ also seem to be a minor effect [65]. It thus appears that giving up the spherical symmetry and spatial uniformity would not have a large impact on the above conclusions.

\section{ACKNOWLEDGMENT}

This work was was supported in part by the U.S. Department of Energy under Grant No. DE-FG02-87ER40371.
[1] D. Kharzeev, Parity violation in hot QCD: Why it can happen, and how to look for it, Phys. Lett. B 633, 260 (2006).

[2] D. Kharzeev and A. Zhitnitsky, Charge separation induced by $P$-odd bubbles in QCD matter, Nucl. Phys. A 797, 67 (2007).

[3] K. Fukushima, D. E. Kharzeev, and H. J. Warringa, The chiral magnetic effect, Phys. Rev. D 78, 074033 (2008).

[4] D. E. Kharzeev, L. D. McLerran, and H. J. Warringa, The effects of topological charge change in heavy ion collisions: "Event by event $P$ and $C P$ violation," Nucl. Phys. A 803, 227 (2008).

[5] D. E. Kharzeev, Topologically induced local $P$ and $C P$ violation in QCD $\times$ QED, Ann. Phys. 325, 205 (2010).

[6] S. L. Adler, Axial vector vertex in spinor electrodynamics, Phys. Rev. 177, 2426 (1969).

[7] J. S. Bell and R. Jackiw, A PCAC puzzle: $\pi^{0} \rightarrow \gamma \gamma$ in the $\sigma$ model, Nuovo Cimento A 60, 47 (1969).

[8] V. Skokov, A. Y. Illarionov, and V. Toneev, Estimate of the magnetic field strength in heavy-ion collisions, Int. J. Mod. Phys. A 24, 5925 (2009).

[9] V. Voronyuk, V. D. Toneev, W. Cassing, E. L. Bratkovskaya, V. P. Konchakovski, and S. A. Voloshin, Electromagnetic field evolution in relativistic heavy-ion collisions, Phys. Rev. C 83, 054911 (2011).

[10] L. Ou and B. A. Li, Magnetic effects in heavy-ion collisions at intermediate energies, Phys. Rev. C 84, 064605 (2011).

[11] A. Bzdak and V. Skokov, Event-by-event fluctuations of magnetic and electric fields in heavy ion collisions, Phys. Lett. B 710, 171 (2012).

[12] J. Bloczynski, X. G. Huang, X. Zhang, and J. Liao, Azimuthally fluctuating magnetic field and its impacts on observables in heavy-ion collisions, Phys. Lett. B 718, 1529 (2013).

[13] W. T. Deng and X. G. Huang, Event-by-event generation of electromagnetic fields in heavy-ion collisions, Phys. Rev. C 85, 044907 (2012).

[14] E. Stewart and K. Tuchin, Magnetic field in expanding quarkgluon plasma, Phys. Rev. C 97, 044906 (2018).

[15] B. Peroutka and K. Tuchin, Quantum diffusion of electromagnetic fields of ultrarelativistic spin-half particles, Nucl. Phys. A 966, 64 (2017).

[16] K. Tuchin, Synchrotron radiation by fast fermions in heavy-ion collisions, Phys. Rev. C 82, 034904 (2010); 83, 039903(E) (2011).

[17] K. Tuchin, Initial value problem for magnetic fields in heavy ion collisions, Phys. Rev. C 93, 014905 (2016).

[18] Y. Hirono, T. Hirano, and D. E. Kharzeev, The chiral magnetic effect in heavy-ion collisions from event-by-event anomalous hydrodynamics, arXiv:1412.0311.

[19] Y. Yin and J. Liao, Hydrodynamics with chiral anomaly and charge separation in relativistic heavy ion collisions, Phys. Lett. B 756, 42 (2016).

[20] D. E. Kharzeev and H. J. Warringa, Chiral Magnetic conductivity, Phys. Rev. D 80, 034028 (2009).

[21] D. Bodeker, On the effective dynamics of soft nonAbelian gauge fields at finite temperature, Phys. Lett. B 426, 351 (1998).
[22] P. B. Arnold, D. T. Son, and L. G. Yaffe, Effective dynamics of hot, soft non-Abelian gauge fields: Color conductivity and $\log (1 / \alpha)$ effects, Phys. Rev. D 59, 105020 (1999).

[23] D. Grabowska, D. B. Kaplan, and S. Reddy, Role of the electron mass in damping chiral plasma instability in supernovae and neutron stars, Phys. Rev. D 91, 085035 (2015).

[24] Y. Hirono, D. E. Kharzeev, and Y. Yin, Self-similar inverse cascade of magnetic helicity driven by the chiral anomaly, Phys. Rev. D 92, 125031 (2015).

[25] K. Tuchin, Electromagnetic field and the chiral magnetic effect in the quark-gluon plasma, Phys. Rev. C 91, 064902 (2015).

[26] Y. Hirono, D. E. Kharzeev, and Y. Yin, Quantized Chiral Magnetic Current from Reconnections of Magnetic Flux, Phys. Rev. Lett. 117, 172301 (2016).

[27] K. Tuchin, Excitation of Chandrasekhar-Kendall photons in quark gluon plasma by propagating ultrarelativistic quarks, Phys. Rev. C 93, 054903 (2016).

[28] F. Wilczek, Two Applications of Axion Electrodynamics, Phys. Rev. Lett. 58, 1799 (1987).

[29] S. M. Carroll, G. B. Field, and R. Jackiw, Limits on a Lorentzand parity violating modification of electrodynamics, Phys. Rev. D 41, 1231 (1990).

[30] P. Sikivie, On the interaction of magnetic monopoles with axionic domain walls, Phys. Lett. B 137, 353 (1984).

[31] S. Chandrasekhar, On force-free magnetic fields, Proc. Natl. Acad. Sci. USA 42, 1 (1956); S. Chandrasekhar and P. C. Kendall, On force-free magnetic fields, Astrophys. J. 126, 457 (1957).

[32] D. T. Son and A. R. Zhitnitsky, Quantum anomalies in dense matter, Phys. Rev. D 70, 074018 (2004).

[33] H. U. Yee and Y. Yin, Realistic implementation of chiral magnetic wave in heavy ion collisions, Phys. Rev. C 89, 044909 (2014).

[34] K. Tuchin, Particle production in strong electromagnetic fields in relativistic heavy-ion collisions, Adv. High Energy Phys. 2013, 490495 (2013).

[35] K. Tuchin, Time and space dependence of the electromagnetic field in relativistic heavy-ion collisions, Phys. Rev. C 88, 024911 (2013).

[36] B. G. Zakharov, Electromagnetic response of quark-gluon plasma in heavy-ion collisions, Phys. Lett. B 737, 262 (2014).

[37] G. Aarts, C. Allton, J. Foley, S. Hands, and S. Kim, Spectral Functions at Small Energies and the Electrical Conductivity in Hot, Quenched Lattice QCD, Phys. Rev. Lett. 99, 022002 (2007)

[38] H.-T. Ding, A. Francis, O. Kaczmarek, F. Karsch, E. Laermann, and W. Soeldner, Thermal dilepton rate and electrical conductivity: An analysis of vector current correlation functions in quenched lattice QCD, Phys. Rev. D 83, 034504 (2011).

[39] G. Aarts, C. Allton, A. Amato, P. Giudice, S. Hands, and J. I. Skullerud, Electrical conductivity and charge diffusion in thermal QCD from the lattice, J. High Energy Phys. 02 (2015) 186. 
[40] W. Cassing, O. Linnyk, T. Steinert, and V. Ozvenchuk, On the Electric Conductivity of Hot QCD Matter, Phys. Rev. Lett. 110, 182301 (2013).

[41] Y. Yin, Electrical conductivity of the quark-gluon plasma and soft photon spectrum in heavy-ion collisions, Phys. Rev. C 90, 044903 (2014).

[42] M. Joyce and M. E. Shaposhnikov, Primordial Magnetic Fields, Right-Handed Electrons, and the Abelian Anomaly, Phys. Rev. Lett. 79, 1193 (1997).

[43] A. Boyarsky, J. Frohlich, and O. Ruchayskiy, Self-Consistent Evolution of Magnetic Fields and Chiral Asymmetry in the Early Universe, Phys. Rev. Lett. 108, 031301 (2012).

[44] H. Tashiro, T. Vachaspati, and A. Vilenkin, Chiral effects and cosmic magnetic fields, Phys. Rev. D 86, 105033 (2012).

[45] I. Rogachevskii, O. Ruchayskiy, A. Boyarsky, J. Fröhlich, N. Kleeorin, A. Brandenburg, and J. Schober, Laminar and turbulent dynamos in chiral magnetohydrodynamics. I. Theory, Astrophys. J. 846, 153 (2017).

[46] P. Pavlovic, N. Leite, and G. Sigl, Chiral magnetohydrodynamic turbulence, Phys. Rev. D 96, 023504 (2017).

[47] N. Yamamoto, Scaling laws in chiral hydrodynamic turbulence, Phys. Rev. D 93, 125016 (2016).

[48] X.-1. Xia, H. Qin, and Q. Wang, Approach to ChandrasekharKendall-Woltjer state in a chiral plasma, Phys. Rev. D 94, 054042 (2016).

[49] C. Manuel and J. M. Torres-Rincon, Dynamical evolution of the chiral magnetic effect: Applications to the quark-gluon plasma, Phys. Rev. D 92, 074018 (2015).

[50] D. E. Kharzeev, The chiral magnetic effect and anomaly-induced transport, Prog. Part. Nucl. Phys. 75, 133 (2014).

[51] Z. V. Khaidukov, V. P. Kirilin, A. V. Sadofyev, and V. I. Zakharov, On magnetostatics of chiral media, arXiv:1307.0138.

[52] V. P. Kirilin, A. V. Sadofyev, and V. I. Zakharov, Anomaly and long-range forces, arXiv:1312.0895.
[53] A. Avdoshkin, V. P. Kirilin, A. V. Sadofyev, and V. I. Zakharov, On consistency of hydrodynamic approximation for chiral media, Phys. Lett. B 755, 1 (2016).

[54] Y. Akamatsu and N. Yamamoto, Chiral Plasma Instabilities, Phys. Rev. Lett. 111, 052002 (2013).

[55] M. Dvornikov and V. B. Semikoz, Magnetic field instability in a neutron star driven by the electroweak electron-nucleon interaction versus the chiral magnetic effect, Phys. Rev. D 91, 061301 (2015).

[56] P. V. Buividovich and M. V. Ulybyshev, Numerical study of chiral plasma instability within the classical statistical field theory approach, Phys. Rev. D 94, 025009 (2016).

[57] G. Sigl and N. Leite, Chiral magnetic effect in protoneutron stars and magnetic field spectral evolution, J. Cosmol. Astropart. Phys. (2016), 025 (2016).

[58] V. P. Kirilin and A. V. Sadofyev, Anomalous transport and generalized axial charge, Phys. Rev. D 96, 016019 (2017).

[59] D. Biskamp, Nonlinear Magnetohydrodynamics (Cambridge University Press, Cambridge, England, 1993).

[60] K. Tuchin, Taming instability of magnetic field in chiral medium, Nucl. Phys. A 969, 1 (2018).

[61] D. B. Kaplan, S. Reddy, and S. Sen, Energy conservation and the chiral magnetic effect, Phys. Rev. D 96, 016008 (2017).

[62] A. R. Zhitnitsky, P odd fluctuations and long range order in heavy ion collisions: Deformed QCD as a toy model, Nucl. Phys. A 897, 93 (2013).

[63] E. Thomas and A. R. Zhitnitsky, Long range order in gauge theories: Deformed QCD as a toy model, Phys. Rev. D 87, 085027 (2013).

[64] M. N. Chernodub, Free magnetized knots of parity-violating deconfined matter in heavy-ion collisions, arXiv:1002.1473.

[65] K. Tuchin, Spontaneous topological transitions of electromagnetic fields in spatially inhomogeneous $C P$-odd domains, Phys. Rev. C 94, 064909 (2016). 